\title{
A time domain beam propagation method for chirped signals
}

\author{
Stefan Jakobsson, $\quad$ Elin Solberg, $\quad$ Fredrik Edelvik, \\ Fraunhofer-Chalmers Research Centre for Industrial Mathematics, \\ Chalmers Science Park, SE-412 88 Gothenburg, Sweden \\ E-mail: \\ stefan.jakobsson@fcc.chalmers.se, elin.solberg@fcc.chalmers.se, fredrik.edelvik@fcc.chalmers.se
}

\begin{abstract}
Chirped pulses are used in time stretch analog to digital converters to stretch high speed electric signals so that they can be measured with conventional detectors. Here a time domain beam propagation method for strongly chirped signals propagating along a specified direction is presented. By examining the propagation of chirped pulses in waveguides we derive a phase factor which captures the rapidly oscillating part of the chirped pulse. We then solve for the slowly varying envelope with respect to this phase factor in a time window moving with the pulse. The new method is applied to simulation of electro-optical modulators for chirped pulses with promising results.
\end{abstract}

Keywords: Time domain beam propagation, finite elements, chirped signals, time stretch systems

\section{Introduction}

The Beam Propagation Method (BPM) [1, p. 329ff.] is used in optical and acoustical wave propagation for solving the Helmholtz equation when there is an assumed propagation direction and a specified frequency (wavenumber). It employs the Slowly Varying Envelope Approximation (SVEA) to factor out a fast varying phase function. For propagation along the $z$ axis it takes the form

$$
\phi(\mathbf{x})=\exp (-j \beta z) \psi(\mathbf{x}),
$$

where $\beta$ is a method parameter to be chosen appropriately. This method has been extended to time domain, TD-BPM, to simulate pulses propagating in optical devices. In a series of papers Masoudi et al have developed time domain BPM with a moving time window following the pulse with the group velocity [2, 3, 4]. In their approach a pulse traveling along the positive $z$ direction with mean angular frequency $\omega_{0}$ is written

$$
\phi(\mathbf{x}, t)=\psi(x, y, z, t-z / v) \exp \left(j\left(\omega_{0} t-\beta z\right)\right),
$$

where $v$ is an estimate of the pulse velocity and the variable $\tau=t-z / v$ is called the moving time and will replace the ordinary time variable in the computations.

An ultrashort optical pulse is an electromagnetic pulse with duration less than a pico second and down to a femto second [5]. Such pulses are broadband in spectrum and when they travel through a dispersive optical fiber they are transformed into chirped pulses of much longer time duration (the time stretch can be of order 10000 or more). Since different frequencies travel with different speeds in a dispersive fiber this will implicitly induce a time to wavenumber mapping which is important for example for time stretched analog to digital converters [6]. The phase factor in Equation (1) will then not capture the oscillation of the chirped signal and therefore the approach in its current state will not work for this case. After the dispersive fiber, the chirped signal is guided into other optical devices. Here our interest is to study propagation in electro-optical modulators [7, pp. 2-3]. 
In this work we extend the TD-BPM to strongly chirped pulses. This is accomplished by utilizing a modified phase factor which takes into account that the frequency varies in time and space for chirped signals. The specific form of this phase factor is derived by analyzing propagation of chirped pulses in optical waveguides by means of the method of stationary phase.

We illustrate the method by applying it to simulations of electro-optical modulators with fast electric signals.

\section{Pulses in waveguides}

In this section we analyze pulses travelling in waveguides by means of the Fourier transform and the method of stationary phase. We first analyze propagation of pulses without chirp, and see that such analysis motivates the choice of phase factor made by Masoudi et al for their TD-BPM in [2, 3, 4]. We then investigate the generation of chirped pulses, before we analyze the propagation of chirped pulses in waveguides, in order to find a suitable phase factor for that case.

\subsection{Propagation of non-chirped pulses}

Propagation along the $z$-direction in an optical waveguide can be characterized by a frequency dependent propagation constant $\beta(\omega)$ and a mode shape $M(x, y, \omega)$, which can both be obtained by solving a mode problem in the cross section. Let $\phi_{0}(t)$ be the initial complex intensity function whose frequency content is centered around the angular frequency $\omega_{0}$. Given this, we can write the propagation of this pulse in the waveguide

$$
\phi(z, t)=\frac{1}{2 \pi} \int \exp (j(\omega t-\beta(\omega) z)) \widehat{\phi}_{0}(\omega) d \omega,
$$

where $\widehat{\phi}_{0}$ is the Fourier transform of $\phi_{0}$. Expand the propagation constant in Taylor series around $\omega_{0}$,

$$
\beta(\omega)=\beta\left(\omega_{0}\right)+\left(\omega-\omega_{0}\right) \beta^{\prime}\left(\omega_{0}\right)+\frac{1}{2}\left(\omega-\omega_{0}\right)^{2} B(\omega) .
$$

For future reference we introduce the phase index $n_{p}$ (inverse of phase velocity) and the group index $n_{g}$ (inverse of group velocity) as

$$
n_{p}=\frac{\beta\left(\omega_{0}\right)}{\omega_{0}}, \quad n_{g}=\beta^{\prime}\left(\omega_{0}\right)
$$

The phase in the Fourier integral (2) can now be written

$$
\omega t-\beta(\omega) z=\left(\omega_{0} t-\beta\left(\omega_{0}\right) z\right)+\left(\omega-\omega_{0}\right)\left(t-\beta^{\prime}\left(\omega_{0}\right) z\right)-\frac{1}{2}\left(\omega-\omega_{0}\right)^{2} B(\omega) z .
$$

Inserting into (2) and using the definition of the inverse Fourier transform yields

$$
\phi(z, t)=\exp \left(j\left(\omega_{0} t-\beta\left(\omega_{0}\right) z\right)\right) \mathcal{F}^{-1}\left[\exp \left(-j \frac{1}{2} \omega^{2} B\left(\omega+\omega_{0}\right) z\right) \widehat{\phi}_{0}\left(\omega+\omega_{0}\right)\right]\left(t-\beta^{\prime}\left(\omega_{0}\right) z\right)
$$

This can be compared with the decomposition in (1) into an explicit fast varying phase function and a slowly varying envelope moving with the group velocity.

\subsection{Creation of chirped pulses}

Chirped pulses are generated by letting pulses pass through dispersive optical fibers, which can be viewed as long waveguides, hence (2) applies. If we neglect non-linear effects on the fiber, the Fourier transform of the signal after leaving the fiber is then $\exp \left(-j \beta_{\mathrm{f}}(\omega) L_{\mathrm{f}}\right) \widehat{\phi_{0}}(\omega)$, where 
$\beta_{\mathrm{f}}$ and $L_{\mathrm{f}}$ are the propagation constant and length, respectively, of the dispersive fiber, and $\phi_{0}$ is the signal entering the fiber.

In the next section we analyze the propagation of chirped pulses through waveguides, so that the signal leaving the fiber is the one entering the waveguide. To simplify notation we let $\kappa(\omega)=\beta_{\mathrm{f}}(\omega) L_{\mathrm{f}}$. From (3) it follows that the zeroth and first order terms of $\beta_{\mathrm{f}}$ (and hence of $\kappa)$ correspond merely to a phase shift and translation in time, respectively, of $\phi_{0}$, which may be ignored in the following analysis. We can then write, for linearly chirped signals,

$$
\kappa(\omega)=\frac{1}{2} \kappa_{2}\left(\omega-\omega_{0}\right)^{2} .
$$

\subsection{Propagation of chirped pulses}

Th beam propagation method works well when the material parameters of the optical device change slowly along the propagation direction. In this sense it can be considered as a weakly disturbed waveguide. To derive an appropriate BPM approach for chirped signals we assume that the signal $\phi_{0}$ enters first a fiber to generate chirp and then a waveguide characterized by a propagation constant $\beta(\omega)$. Then according to Equation (2), the complex amplitude can be written as an oscillatory integral

$$
\phi(z, t)=\frac{1}{2 \pi} \int \exp (j \theta(z, t, \omega)) \widehat{\phi}_{0}(\omega) d \omega
$$

with

$$
\theta(z, t, \omega)=\omega t-\beta(\omega) z-\kappa(\omega) .
$$

To analyze this we need the following well known result [8].

Theorem 1 (Method of stationary phase in one dimension). Consider the one dimensional integral

$$
I=\int_{-\infty}^{\infty} F(\omega) \exp (j \theta(\omega)) d \omega
$$

where $\theta$ is a rapidly varying function relative $F$. Assume $\theta$ has one stationary point $\omega_{s}$

$$
\theta^{\prime}\left(\omega_{s}\right)=0
$$

Then

$$
I \approx \sqrt{\frac{2 \pi}{j \theta^{\prime \prime}\left(\omega_{s}\right)}} F\left(\omega_{s}\right) \exp \left(j \theta\left(\omega_{s}\right)\right) .
$$

According to the theorem of stationary phase the major contribution comes from where the derivative of the phase function is zero

$$
0=\theta_{\omega}^{\prime}(z, t, \omega)=t-\beta^{\prime}(\omega) z-\kappa^{\prime}(\omega) .
$$

This defines implicitly the stationary wavenumber $\omega_{s}=\omega_{s}(z, t)$ as a function of the time and the $z$-coordinate. For $z=0$, this yields the time to wavenumber mapping mentioned in the introduction. This is implicitly defined by

$$
t=\kappa^{\prime}(\omega(t))
$$

According to the one dimensional version of the method of stationary phase we approximately have

$$
\phi(z, t) \approx \frac{1}{\sqrt{-2 \pi j\left(\beta^{\prime \prime}\left(\omega_{s}\right) z+\kappa^{\prime \prime}\left(\omega_{s}\right)\right)}} \exp \left(j\left(\omega_{s} t-\beta\left(\omega_{s}\right) z-\kappa\left(\omega_{s}\right)\right) \widehat{\phi}_{0}\left(\omega_{s}\right),\right.
$$


where $\omega_{s}=\omega_{s}(z, t)$. This formula is our motivation for the TD-BPM method for propagation of chirped signals to be presented in the next section. Central to this is the phase function defined by

$$
\nu(z, t)=\omega_{s}(z, t) t-\beta\left(\omega_{s}(z, t)\right) z-\kappa\left(\omega_{s}(z, t)\right) .
$$

We now aim to compute this for low order Taylor approximations of $\beta(\omega)$ and $\kappa(\omega)$. We note that for chirp generation a fiber of length of kilometer order is used, while the length of the waveguides (or optical devices) of interest is of centimeter order. Due to the short distance it suffices to expand the waveguide propagation coefficient to first order,

$$
\beta(\omega)=\beta\left(\omega_{0}\right)+\beta^{\prime}\left(\omega_{0}\right)\left(\omega-\omega_{0}\right)=\omega_{0} n_{p}+n_{g}\left(\omega-\omega_{0}\right) .
$$

Inserting this and $\kappa$ from (4) into relation (5) for the stationary wavenumber and solving for $\omega_{s}$ we get

$$
\omega_{s}=\omega_{0}+\frac{t-n_{g} z}{\kappa_{2}}=\omega_{0}+\frac{\tau}{\kappa_{2}} .
$$

The expression for $\nu$ then, after simplification, becomes

$$
\nu(z, \tau)=\omega_{0} \tau+\omega_{0}\left(n_{g}-n_{p}\right) z+\frac{\tau^{2}}{2 \kappa_{2}} .
$$

The phase factor for the TD-BPM developed in [2, 3, 4] uses the two first terms of this expression $\nu(z, \tau)=\omega_{0} \tau+\omega\left(n_{g}-n_{p}\right) z$. It is the last term that takes the chirping of the signal into account.

\section{$3 \quad$ Time domain BPM for chirped signals}

The wave equation for a space dependent permittivity $\varepsilon(\mathbf{x}, z)$ is

$$
\Delta_{t} \phi+\partial_{z}^{2} \phi-\varepsilon(\mathbf{x}, z) \partial_{t}^{2} \phi=0
$$

where we have split the Laplacian operator into a transversal and a $z$ dependent part $\Delta=\Delta_{t}+\partial_{z}^{2}$. The moving time window is implemented by a change of variables. We use the moving time $\tau$, defined as

$$
\tau=t-n_{g} z
$$

For clarity we also make the substitution $\zeta=z$. By the chain rule we have

$$
\begin{aligned}
\partial_{z} & =\partial_{\tau} \frac{\partial \tau}{\partial z}+\partial_{\zeta} \frac{\partial \zeta}{\partial z}=\partial_{\zeta}-n_{g} \partial_{\tau} \\
\partial_{t} & =\partial_{\tau} \frac{\partial \tau}{\partial t}+\partial_{\zeta} \frac{\partial \zeta}{\partial t}=\partial_{\tau} .
\end{aligned}
$$

Then

$$
\begin{aligned}
& \partial_{z}^{2}=\partial_{\zeta}^{2}-2 n_{g} \partial_{\zeta} \partial_{\tau}+n_{g}^{2} \partial_{\tau}^{2}, \\
& \partial_{t}^{2}=\partial_{\tau}^{2}
\end{aligned}
$$

In the moving time window coordinates, the wave equation becomes

$$
\partial_{\zeta}^{2} \phi-2 n_{g} \partial_{\zeta} \partial_{\tau} \phi=\left(\varepsilon-n_{g}^{2}\right) \partial_{\tau}^{2} \phi-\Delta_{t} \phi
$$

where we have collected all terms including derivatives with respect to $\zeta$ on the left hand side.

Remark 1. Be aware of that although $\zeta=z$, the partial derivatives with respect to these variables do not coincide. Indeed it seems as though the mixed derivative term $2 n_{g} \partial_{\zeta} \partial_{\tau}$ is missing in the works of Masoudi et al [2, 3, 4]. 
For the derivation we assume the slowly varying envelope approximation

$$
\phi(\mathbf{x}, z, t)=\exp (j \nu(\zeta, \tau)) \psi(\mathbf{x}, \zeta, \tau),
$$

with the phase factor $\exp (j \nu(\zeta, \tau))$ and slowly varying envelope $\psi$. The second order derivatives of $\phi$ become

$$
\begin{aligned}
\partial_{\zeta}^{2} \phi & =\exp (j \nu)\left(\partial_{\zeta}^{2} \psi+2 j \partial_{\zeta} \nu \partial_{\zeta} \psi+\left(j \partial_{\zeta}^{2} \nu-\left(\partial_{\zeta} \nu\right)^{2}\right) \psi\right) \\
\partial_{\zeta} \partial_{\tau} \phi & =\exp (j \nu)\left(\partial_{\zeta} \partial_{\tau} \psi+j \partial_{\zeta} \nu \partial_{\tau} \psi+j \partial_{\tau} \nu \partial_{\zeta} \psi+\left(j \partial_{\zeta} \partial_{\tau} \nu-\partial_{\zeta} \nu \partial_{\tau} \nu\right) \psi\right), \\
\partial_{\tau}^{2} \phi & =\exp (j \nu)\left(\partial_{\tau}^{2} \psi+2 j \partial_{\tau} \nu \partial_{\tau} \psi+\left(j \partial_{\tau}^{2} \nu-\left(\partial_{\tau} \nu\right)^{2}\right) \psi\right) .
\end{aligned}
$$

Collect terms in the wave equation for $\psi$ and divide by $\exp (j \nu(\zeta, \tau))$ to get

$$
\partial_{\zeta}^{2} \psi-2 n_{g} \partial_{\tau} \partial_{\zeta} \psi+a \partial_{\zeta} \psi=\left(\varepsilon-n_{g}^{2}\right) \partial_{\tau}^{2} \psi+\left(b+c\left(\varepsilon-n_{g}^{2}\right)\right) \partial_{\tau} \psi+\left(d+e\left(\varepsilon-n_{g}^{2}\right)\right) \psi-\Delta_{t} \psi .
$$

All coefficients here are functions

$$
\begin{aligned}
a & =2 j\left[\partial_{\zeta} \nu-n_{g} \partial_{\tau} \nu\right], \\
b & =2 j n_{g} \partial_{\zeta} \nu \\
c & =2 j \partial_{\tau} \nu \\
d & =2 n_{g}\left(j \partial_{\zeta} \partial_{\tau} \nu-\partial_{\zeta} \nu \partial_{\tau} \nu\right)-\left(j \partial_{\zeta}^{2} \nu-\left(\partial_{\zeta} \nu\right)^{2}\right), \\
e & =j \partial_{\tau}^{2} \nu-\left(\partial_{\tau} \nu\right)^{2} .
\end{aligned}
$$

In the paraxial approximation, the first two terms on the left hand side of (7) are omitted. We can write

$$
a \partial_{\zeta} \psi=\left(\varepsilon-n_{g}^{2}\right) \partial_{\tau}^{2} \psi+\left(b+c\left(\varepsilon-n_{g}^{2}\right)\right) \partial_{\tau} \psi+\left(d+e\left(\varepsilon-n_{g}^{2}\right)\right) \psi-\Delta_{t} \psi .
$$

Inspired by the analysis in the previous section we suggest that for chirped pulses, choosing $\nu$ as in (6) is appropriate.

\section{Numerical examples}

In this section we will study the propagation of a chirped optical pulse in an electro-optical modulator with co-propagating modulating electric signal. The electric signal causes a shift in the permittivity which affects the optical pulse. We begin by defining the moving time window and then extend the initial pulse to this window.

\subsection{Initial signal}

Maybe the simplest chirped pulse is the linearly chirped Gaussian signal which takes the form

$$
\phi_{\text {chirp }}(t)=\frac{1}{\sqrt{2 \pi} \sigma_{\text {chirp }}} \exp \left(-\frac{t^{2}}{2 \sigma_{\text {chirp }}^{2}}\right) \exp \left(j \omega_{0} t\right)
$$

where

$$
\sigma_{\text {chirp }}=\sqrt{\sigma^{2}+j \widehat{\beta_{2}} L}=\sqrt{\sigma^{2}+j \kappa_{2}}
$$

Note that $\sigma_{\text {chirp }}$ is complex and it is this fact which causes the chirp.

Let $\Omega_{\text {cross }}$ be the cross section of the geometry normal to the propagation direction. The moving time window is the product space $\Omega_{\text {cross }} \times[-T, T]$ where $T$ is chosen so large that it by a wide margin covers the interesting part of $(8)$. Let $M(\cdot, \omega)$ be the frequency dependent mode 
solution for the initial permittivity $\varepsilon\left(\mathbf{x}, z_{0}\right)$. A reasonable initial signal defined in the whole time window is then

$$
\phi_{0}(\mathbf{x}, t)=\phi_{\operatorname{chirp}}(t) M(\mathbf{x}, \omega(t)) .
$$

Since the frequency varies in time as described by the time to wavenumber mapping we can take the dependence of mode shape on time into account (a simplified version would be $\phi_{0}(\mathbf{x}, t)=$ $\phi_{\text {chirp }}(t) M\left(\mathbf{x}, \omega_{0}\right)$, where $\omega_{0}$ is the mean frequency). The initial function $\psi_{0}$ can be obtained from $\phi_{0}$ by factoring out the phase factor $\exp (j \nu(0, t))$.

Remark 2. We now check the accuracy of the phase estimate (6) for a chirped Gaussian signal. The relative error compared to the phase for the true function is

$$
\left|\frac{\arg \left(\phi_{0}(\mathbf{x}, t)\right)-\nu(0, t)}{\arg \left(\phi_{0}(\mathbf{x}, t)\right)}\right|=\frac{\sigma^{4}}{\kappa_{2}^{2}} .
$$

Hence we have a very good initial phase estimate whenever $\sigma$ is small, as it will be in the interesting cases. We note that the error grows with decreasing $\kappa_{2}$. This is because the method of stationary phase is not applicable to non-chirped pulses.

\subsection{Simulation of electro-optical modulator}

The method has been implemented with finite elements, using FEniCS [9], in the moving time window $\Omega_{\text {cross }} \times[-T, T]$ and a Crank-Nicolson scheme for stepping in the $z$ direction. The moving time window is chosen large enough that, for first simulation results, homogeneous Neumann conditions may be used. In the implementation, the term $\varepsilon \partial_{\tau}^{2} \psi$ has been replaced by $\partial_{\tau} \varepsilon \partial_{\tau} \psi$, for easier implementation of the weak formulation. This may be justified considering the small time derivative of $\varepsilon$.

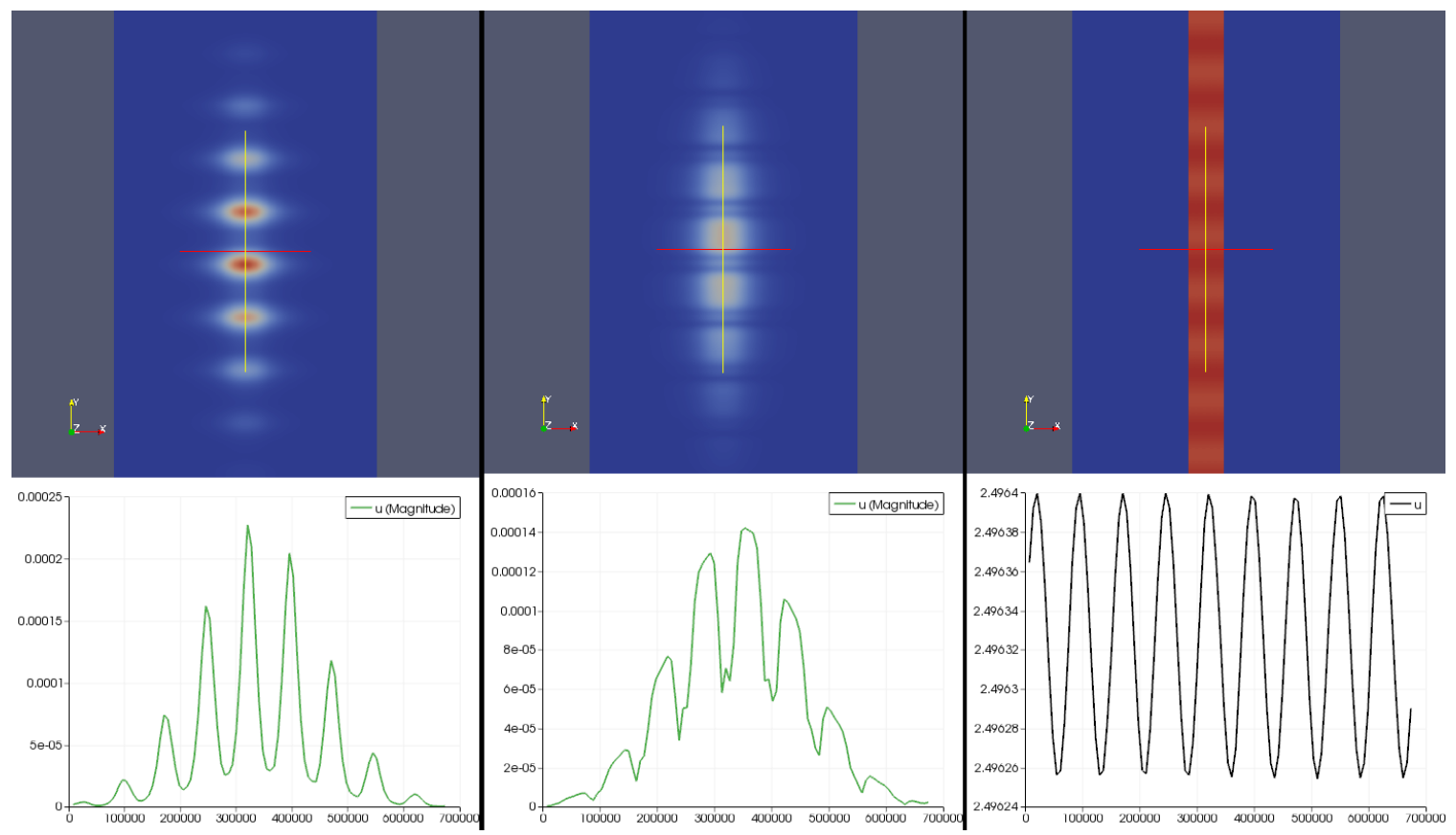

Figure 1: The upper left and the upper middle subfigures show the absolute values of the sum and the difference, respectively, of the modulated and un-modulated signal, and the upper right figure shows the sine shaped modulated permittivity. The lower subfigures are line plots of the corresponding quantities along the central axis.

We now apply the method to simulation of electro-optical modulators. For each case two simulations are performed: one with constant permittivity and the other with a shifted permittivity moving along with the pulse, corresponding to the unmodulated and modulated arm of 
the modulator, respectively. The results from the shifted and un-shifted simulations are added and subtracted

$$
\psi_{\text {add }}=\psi_{\text {shift }}+\psi_{\text {no-shift }}, \quad \psi_{\text {sub }}=\psi_{\text {shift }}-\psi_{\text {no-shift }} .
$$

We consider a case of sine shaped shifted permittivity with maximum amplitude $V_{\pi}$, the voltage causing a half wavelength shift of the signal for the mean frequency $f_{0}$. At maximum signal amplitude (corresponding to minimum permittivity) there should thus be cancellation in $\psi_{\text {add }}$. The initial chirped pulse is chosen as described in the previous section, with the following parameters: $\sigma=1.37 \cdot 10^{-13} \mathrm{~s}, \kappa_{2}=-5.10 \cdot 10^{-23} \mathrm{~s}^{2}, \omega_{0}=1.22 \cdot 10^{15} \mathrm{~s}^{-1}$.

Figure 1 shows the absolute values of $\psi_{\text {add }}$ and $\psi_{\text {sub }}$ at the end of the modulator. Modulation is not perfect, but there is a clear tendency towards the expected cancellation in both $\psi_{\text {add }}$ and $\psi_{\text {sub }}$

\section{Conclusion}

We have developed an extension of the time-domain beam propagation method for strongly chirped signals. An appropriate phase factor was derived by analyzing propagation of chirped pulses in waveguides by means of the Fourier transform and the method of stationary phase. The method has been implemented and first simulations have been performed for electro-optical modulators. The results are promising, but imperfections in the modulation will be investigated further.

\section{References}

[1] K. Okamoto. Fundamentals of Optical Waveguides. Elsevier Science, 2010.

[2] Husain M. Masoudi. A novel nonparaxial time-domain beam-propagation method for modeling ultrashort pulses in optical structures. J. Lightwave Technol., 25(10):3175-3184, Oct 2007 .

[3] H.M. Masoudi and M.S. Akond. Efficient iterative time-domain beam propagation methods for ultra short pulse propagation: Analysis and assessment. Lightwave Technology, Journal of, 29(16):2475 -2481, aug.15, 2011.

[4] H.M. Masoudi and M.S. Akond. Stable time-domain beam propagation method for modeling ultrashort pulse propagation in dispersive optical structures. Photonics Technology Letters, IEEE, 24(9):769 -771, may1, 2012.

[5] A. Weiner. Ultrafast Optics. Wiley Series in Pure and Applied Optics. Wiley, 2011.

[6] Wikipedia. Time stretch analog-to-digital converter - Wikipedia, the free encyclopedia, 2013. [Online; accessed 28-December-2013].

[7] Shashi P. Karna, A.T. Yeates, American Chemical Society. Division of Computers in Chemistry, and American Chemical Society. Meeting. Nonlinear optical materials: theory and modeling : developed from a symposium sponsored by the Division of Computers in Chemistry at the 208th National meeting of the American Chemical Society, Washington, DC, August 21-25, 1994. ACS symposium series. American Chemical Society, 1996.

[8] A. Grigis and J. Sjöstrand. Microlocal Analysis for Differential Operators: An Introduction. London Mathematical Society Lecture Note Series. Cambridge University Press, 1994.

[9] A. Logg, K.A. Mardal, and G. Wells. Automated Solution of Differential Equations by the Finite Element Method: The FEniCS Book. Lecture Notes in Computational Science and Engineering. Springer, 2012. 